\title{
Ubiquitous Health Management System with Watch-Type Monitoring Device for Dementia Patients
}

\author{
Dongmin Shin, Dongil Shin, and Dongkyoo Shin \\ Department of Computer Engineering, Sejong University, 98 Gunja-Dong, Gwangjin-Gu, Seoul 143-747, Republic of Korea \\ Correspondence should be addressed to Dongkyoo Shin; shindk@sejong.ac.kr
}

Received 11 November 2013; Revised 13 January 2014; Accepted 19 January 2014; Published 4 March 2014

Academic Editor: Young-Sik Jeong

Copyright (C) 2014 Dongmin Shin et al. This is an open access article distributed under the Creative Commons Attribution License, which permits unrestricted use, distribution, and reproduction in any medium, provided the original work is properly cited.

\begin{abstract}
For patients who have a senile mental disorder such as dementia, the quantity of exercise and amount of sunlight are an important clue for doses and treatment. Therefore, monitoring daily health information is necessary for patients' safety and health. A portable and wearable sensor device and server configuration for monitoring data are needed to provide these services for patients. A watch-type device (smart watch) that patients wear and a server system are developed in this paper. The smart watch developed includes a GPS, accelerometer, and illumination sensor, and can obtain real time health information by measuring the position of patients, quantity of exercise, and amount of sunlight. The server system includes the sensor data analysis algorithm and web server used by the doctor and protector to monitor the sensor data acquired from the smart watch. The proposed data analysis algorithm acquires the exercise information and detects the step count in patients' motion acquired from the acceleration sensor and verifies the three cases of fast pace, slow pace, and walking pace, showing $96 \%$ of the experimental results. If developed and the $\mathrm{u}-$ Healthcare System for dementia patients is applied, higher quality medical services can be provided to patients.
\end{abstract}

\section{Introduction}

The increase in the elderly population due to the development of medical technology is creating challenges for care professionals and developers of ubiquitous healthcare systems.

Dementia refers to the cognitive impairment usually affecting old people and makes functioning in daily life more difficult. Early symptoms of dementia include memory loss gradually affecting everyday activities. Typically from a few months to several years, the first symptoms are mild but develop slowly and gradually lead to serious memory loss. In addition, dementia patients have difficulty in recognizing their family members and doing complicated tasks. They usually have wandering symptoms and more than $73 \%$ experience being lost or missing [1].

The ubiquitous healthcare system is a convergence of information communication technology and healthcare and has emerged in various ways to help these kinds of patients [2]. Keruve, a Spanish company, provides a medical service for dementia patients. This service uses a bracelet with a built-in GPS and a portable device. The GPS bracelet features precise location detection using triangulation, even if the patient is in the room [3]. Korea Telecom, a Korean company, has developed a location-tracking system using GPS and Code Division Multiple Access (CDMA) [4]. Gangnam District Office in Seoul, Korea, has developed a system called Gangnam U-Safe System [5]. This service began in May 2009 using Ubiquitous Sensor Network (USN) technology and GPS. This system provides a compact device featured with an emergency alarm service used for the safety of socially vulnerable individuals including children and those with intellectual disabilities.

Currently, healthcare systems for patients with dementia are focusing on location tracking using a Global Positioning System (GPS). For patients with mental disorders, momentum monitoring and medical service profiling can manage their risks and enhance their quality of life $[6,7]$. In this paper, we develop an ubiquitous health management system for dementia patients to improve their health and safety following the concept Internet of Things (IoT) [8-10]. The system consists of a wrist watch-type device and a server system. The device includes a built-in GPS, ambient light sensor, and acceleration sensor and communicates with the server system. The server system functions include the 
creation of a personal profile for patients and monitoring a patient's location and measuring the amount of sunlight illumination and walking step count to use as medical data. The system helps dementia patients avoid the risk of being missing or lost by wandering symptoms.

\section{Related Works}

Recently, the concept of Internet of Things (IoT) has been applied in ubiquitous healthcare systems and services [8$10]$. Io $\mathrm{T}$ is a novel paradigm of technologies that interconnect everyday objects with each other through the Internet exploiting multiple wireless communication interfaces and advancements in computing devices [11]. With the spread of smart phones and tablets loaded with various sensors such as GPS and accelerometers, higher quality services are provided to the users by connection of the information on the Web and real world [12].

With the advent of IoT, research on numerous medical services for patients has been performed $[9,10]$. Research on wireless networking technologies for developing a mobile healthcare environment has been carried out and it leads into the concept of mobile IoT (m-IoT), which is a new healthcare connectivity paradigm that interconnects IP-based communication technologies such as IPv6 over low power WPAN (6LoWPAN) with emerging $4 \mathrm{G}$ networks for future Internetbased healthcare services [9]. Typically, healthcare services are comprised of the sensors acquiring biosignals and the servers processing the huge amount of biodata generated from the sensors. Service platforms that interconnect cloud computing, distributed processing, and high speed data processing systems following the concept of Io T are being researched for efficient healthcare services [10].

Studies on human movement detection and behavioral patterns have been carried out in various ways for healthcare services. The motion recognition algorithm based on a motion-tree is developed using the acceleration features of a mobile phone [12]. The motion detection algorithm is one of the basic methods for detecting the number of walking steps $[13,14]$. Human movements are distinguished by a pattern recognition algorithm and a way of extracting various motions are developed from basic motion patterns and feature vectors of humans. This function reads normal and abnormal movements, for example, sitting, standing, and falling down, as well as the number of steps [15-18].

Position tracking using GPS is one of the data for measuring the momentum as well as the current position of the patient in a healthcare system. Recently research on indoor position tracking methods using Wi-Fi or other positioning schemes are being carried out because it is impossible to get a GPS signal indoors $[19,20]$.

\section{Development of a Ubiquitous Health Management System}

The system consists of a watch-type monitoring device and server. The monitoring device includes a GPS, 3-axis accelerometer, and ambient light sensor. It is worn on the patient's wrists and periodically transfers his activity information to the server derived from his location and amount of light illumination detecting sun exposure. Then care professionals and doctors can monitor the patient's health condition through the webpage delivered by the server. The server identifies the location through the patient's data transferred from the monitoring device and measures the patient's activity information through the step number detection algorithm and creates a profile about the patient's health information, together with the amount of light illumination to detect sun exposure.

3.1. Development of the Watch-Type Monitoring Devices. In the monitoring device, location-tracing functions using a GPS sensor can monitor the present location and migration route of the patient. The ambient light sensor measures the amount of sunlight illumination exposed to the device and records it. The 3-axis acceleration sensor records the value of the $x$-, $y$-, and $z$-axis coordinate values in real time. The server can get the number of patient's steps through the step detection algorithm.

The values of the sensors are obtained through the real time transfer of the data through Transmission Control Protocol/Internet Protocol (TCP/IP) communication on the CDMA network. After connection to the server through a Short Message Service (SMS) such as Server Open SMS and Transmission Close SMS for transfers, the values of the sensors exchange data with each other. At this moment, the transfer of the data by contacting the server is scheduled according to the regular cycle defined by the user. The server can inform the care professional or patient by alarm in the case of special events such as injection time and escape from patient's safety zone of patient.

The monitoring device is designed to be worn easily using the form factor of a wrist watch and because it is held in position by a clamp, it can prevent a patient from taking it off or losing it. Thus, if a demented patient experiences emergency or wandering symptoms, the problem can be quickly dealt with. The internal block diagram of the watchtype monitoring device proposed in this paper is shown in Figure 1.

3.2. Development of the Health Management Server. The server system is composed of the receiver module for receiving the transmitted data from the monitoring device, the health management module analyzing data, and the webpages performing management functions and patient monitoring, as shown in Figure 2.

First, the receiver module manages the watch's connection through the SMS receiver while waiting for the monitoring device's SMS. The receiver module with the Connection SMS receives the accumulated data saved in the monitoring device as the defined protocols after assigning a socket and a thread using TCP/IP communication.

The health management module generates the patient profile by analyzing the transferred data. It checks whether the user moves out of the scope of the designated safety zone or not using the GPS sensor data. And it converts the ambient 


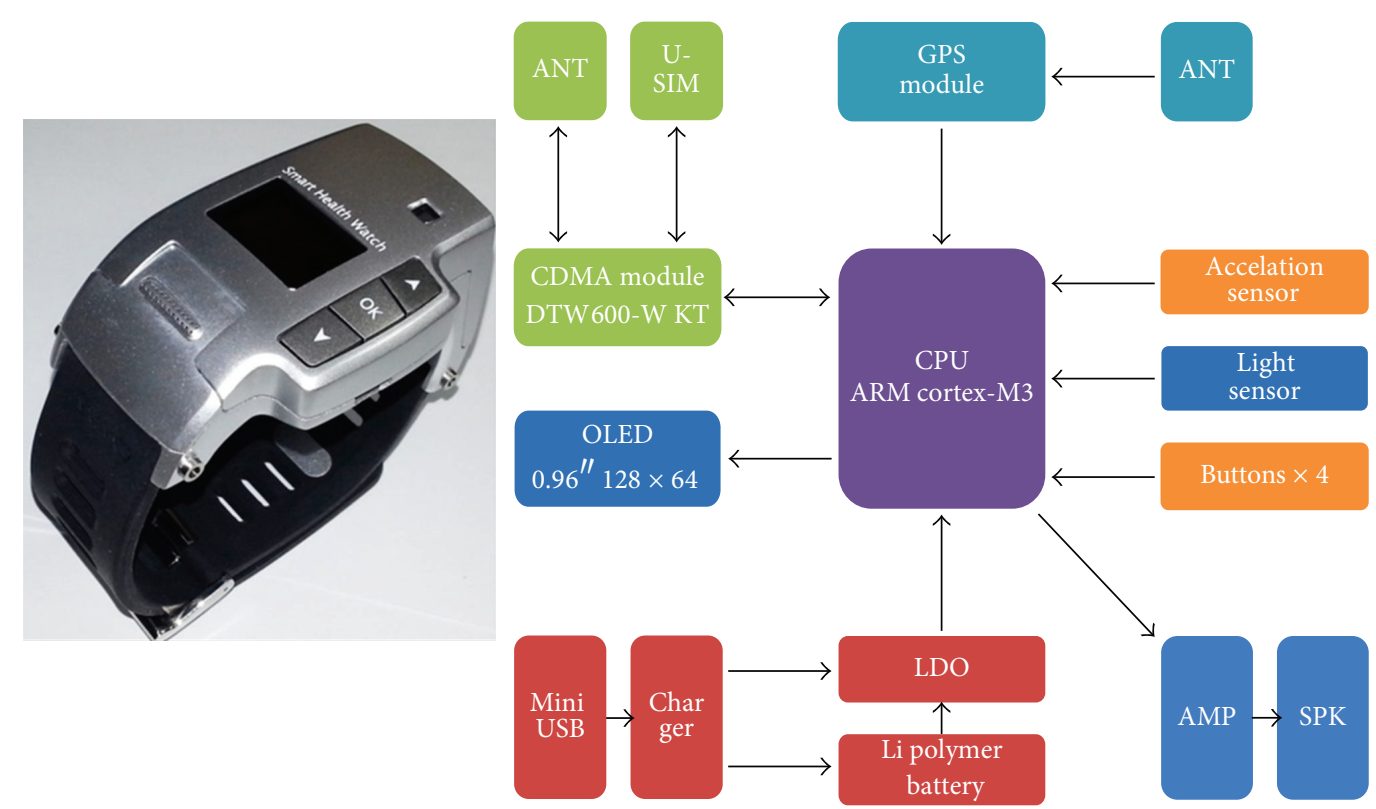

FIgURE 1: Watch-type monitoring device and its internal block diagram.

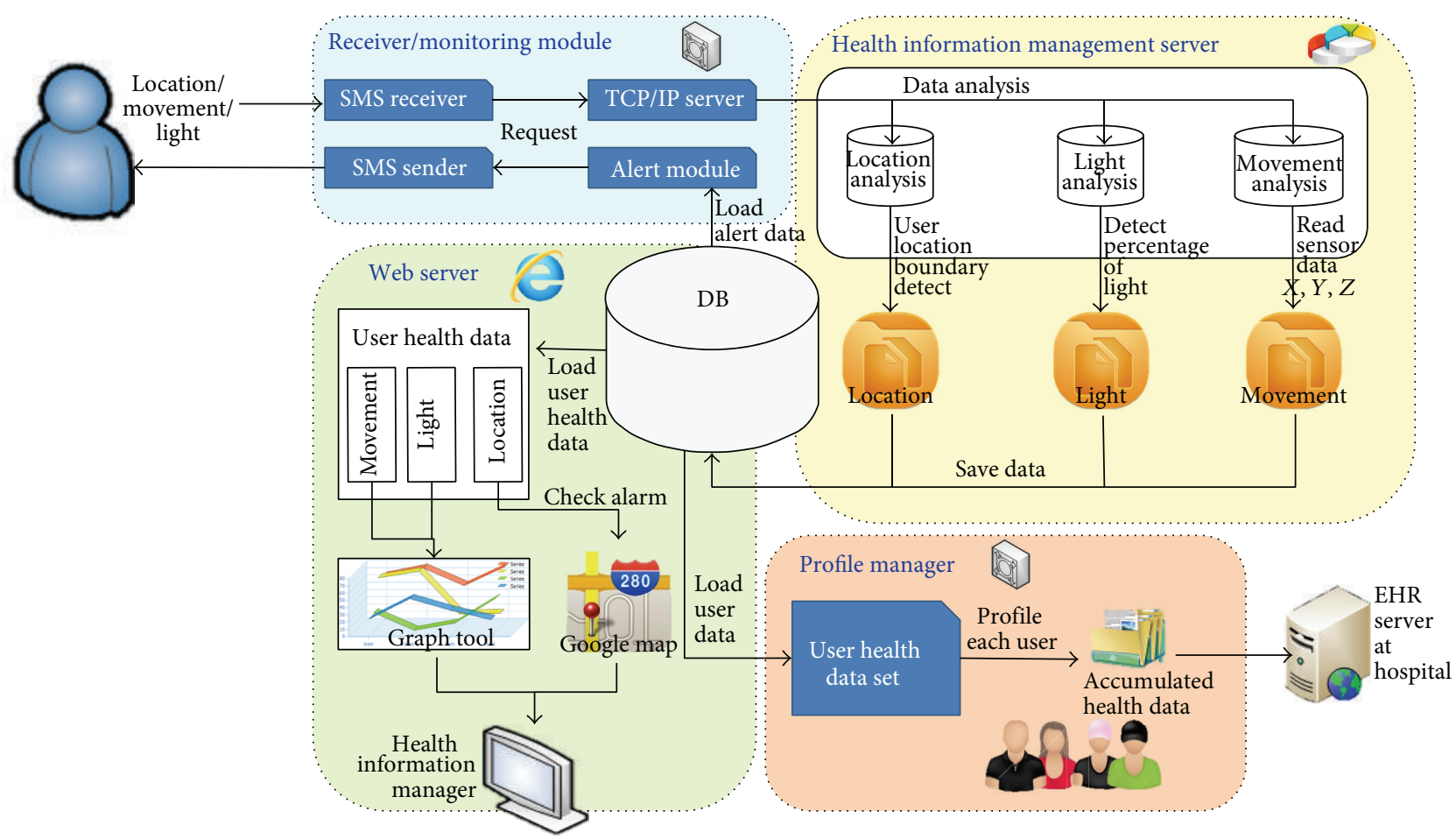

FIGURE 2: The System Operational Scenario.

light sensor data into a percentage from 0 to 100 accounting for the patient's exposure time to sunlight. Finally, it measures the amount of a patient's movement by counting walking steps based on the step detection algorithm using the 3-axis acceleration sensor data. The patient's data acquired from this module is separately saved into the database. The data in the database is used and recorded in the profile of each patient and can be monitored through the webpage.
The webpage is used to monitor the tracing location and health information of the patient obtained from the DB. First of all, a care professional can set up a communication period between the monitoring device and the server and the scope of the safety zone through the settings. The server indicates whether the traced patient's location is within the scope of the safety zone or not, and his present location and the scope of the safety zone would be marked in a circle on 


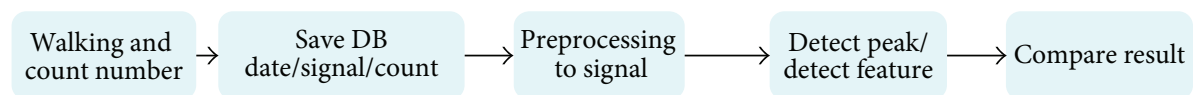

Figure 3: Preprocessing of accelerometer data.

the map. The amount of sunlight indicates the exposure state hourly as the time-axis and exposure-axis through the graph. The activity mass also expresses the number of walk hourly through the graph. The health information can preserve the patient's health and safety because it monitors the patient's state through an activity list by time order, amount of sunlight, and location of the patient measured during outdoor activities.

\section{Walking Step Detection Algorithm}

In addition to the location-tracking service for dementia patients, the system provides accurate walking step detection for use in healthcare. The step detection algorithm uses a 3 -axis accelerometer to accurately detect a patient's steps and further analyzes his activities.

4.1. Experimental Design. The experiment done in this paper uses the watch-type monitoring device to compare the actual steps counted in 30 60 seconds with the value detected by the accelerometer under the same conditions. Eight people took part in this experiment creating 170 data of 3 types of stepsfast steps, normal steps, and slow steps every day. Each data is categorized in the database by experiment date, time, and the number of steps. Stored results are preprocessed into energy values for peak picking and analysis of distinctive features of the walk. Analyzed features are used to distinguish the step and nonstep activities and the measured number of steps is then compared to the actual number of steps counted.

4.2. Preprocessing Data. Figure 3 shows preprocessing of the accelerometer data. Each acquired $x$-, $y$-, $z$-axis data are in 8 byte double data types, recorded 80 times per second. It makes the calculation more efficient using the Signal Vector Magnitude (SVM) values than using 3 values simultaneously for each calculation. SVM in this experiment is expressed as the following equation (see Figure 4)

$$
\mathrm{SVM}=\sqrt{x_{i}^{2}+y_{i}^{2}+z_{i}^{2}}
$$

The accelerometer records 80 times per second and even catches subtle movements. Therefore, even if the patient is standing still, the accelerometer will be recording constantly changing values. These subtle noise signals could result in errors when measuring the number of steps. In this paper, we have used the Moving Average Filter (MAF) to filter out these noises, preventing errors. The MAF has low pass filter properties and it can be expressed as follows:

$$
\begin{aligned}
T[n]=\frac{1}{5}(\operatorname{SVM}[n-2]+\operatorname{SVM}[n-1]+\operatorname{SVM}[n] \\
+\operatorname{SVM}[n+1]+\operatorname{SVM}[n+2]) \\
=\frac{1}{5} \sum_{m=-2}^{2} \operatorname{SVM}[n-m] .
\end{aligned}
$$

Here, the value of $n$th MAF is denoted by $T[n]$ and SVM $[n-$ $1]$ means $(n-1)$ th SVM. Figure 5 shows the result of moving average filter.

4.3. Step Detection Algorithm. The step detection algorithm proposed in this paper finds the peaks from the preprocessed data and then counts the number of peak values that are over the threshold value, which is calculated from the data.

First, to pick out the peaks, we find the wave's mean gradient by computing the average of the gradient of two bundles of data intervals. If this value is greater than the threshold value, it is considered the start of the peak, and when the mean gradient becomes a negative value, this point is put into the peak point candidate. It is expressed as follows:

$$
\begin{gathered}
G_{n}=\frac{\mathrm{SVM}_{n+1}-\mathrm{SVM}_{n}}{T_{n+1}-T_{n}}, \\
\text { Average of } G_{n}=\frac{G_{n}+G_{n+1}}{2} .
\end{gathered}
$$

The peak candidate includes waveform errors or noise errors. The following method is used to clear out the errors and find the genuine peaks. First, we find the peak candidates with a time interval of less than 0.3 seconds. Collected data are acceleration data for detecting the number of steps, so the movements must show regular intervals of high peak and low peak. Therefore, peak candidates in the low period are noise values from the wrong movement. Then, we store the candidate with high SVM values as the actual peak and drop the values considered as errors.

Detected peak values are affected by the patient's footsteps and the height of the swinging of arms, so the values include individual differences. However, every waveform of walking has high amplitude followed by low amplitude. Therefore, we use this feature to derive a threshold value with the mean amplitude over 1 second and collect the peaks over the threshold value. Figure 6 shows the result from the detection of step peaks.

4.4. Results of Experiment. The proposed algorithm is tested with the watch-type monitoring device with an embedded 


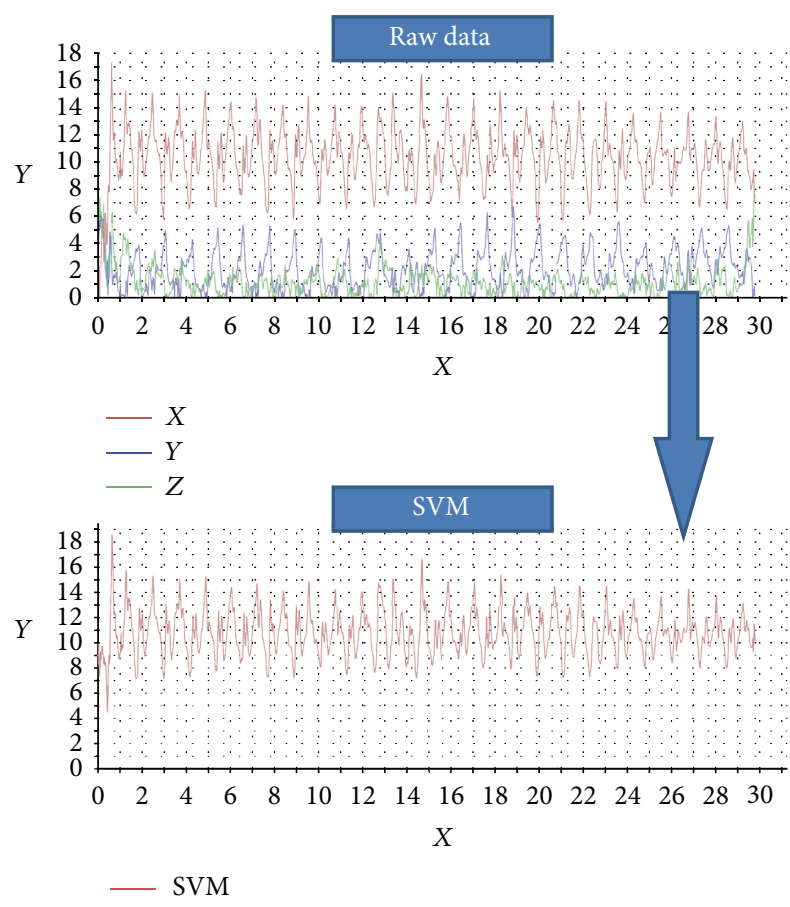

FIgURE 4: Preprocessing: convert raw data to SVM value.

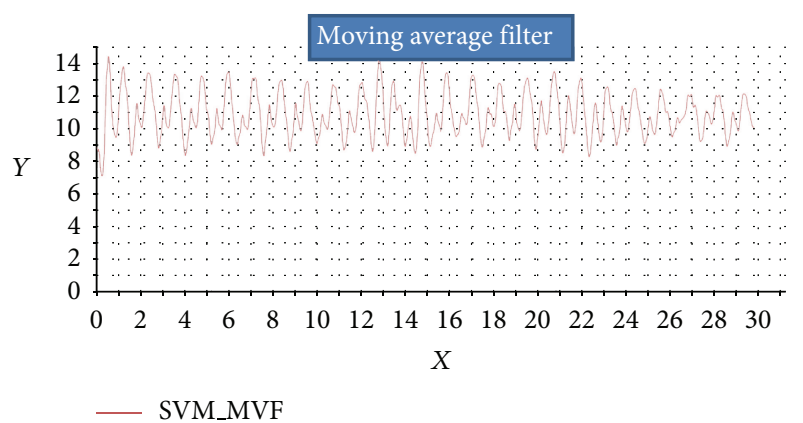

FIGURE 5: Preprocessing: moving average filter.

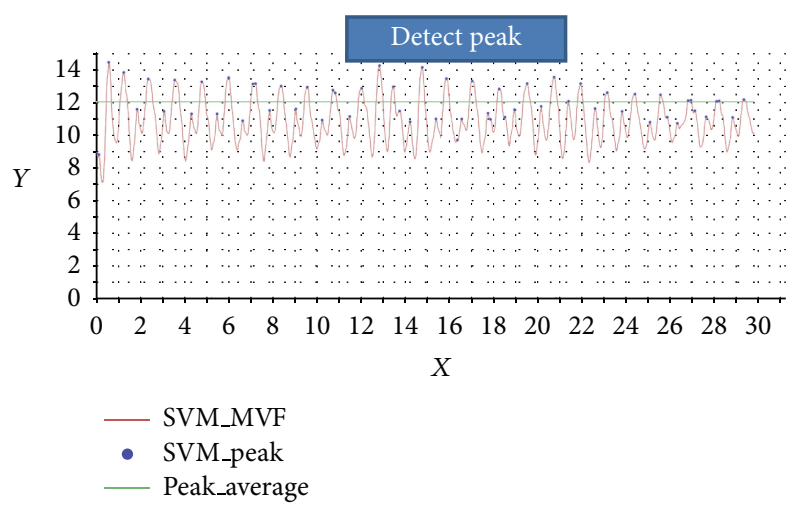

FIGURE 6: Result from the detection of step peaks. accelerometer using an $80 \mathrm{~Hz}$ sample rate, attached to experimenters' wrists, and tested on fast steps, normal steps, and slow steps.

To measure the accuracy of the proposed algorithm, we compared the actual sum of steps and the detected sum of steps derived with the algorithm. The results of this method showed $94.7 \%$ accuracy in total, $93 \%$ in fast steps, $96.7 \%$ in normal steps, and $96 \%$ in slow steps.

As the pace gets faster, the gradient of SVM tends to grow larger and the phase interval narrows, resulting in higher error rates. However, in cases of normal and slow steps in which the amplitude is gradual, results have a higher rate of finding the peaks correctly, showing a closer value to the actual number of steps. Table 1 shows the analyzed data from the 8 people taking part in the experiment.

\section{Patient Profile Management System}

The purpose of this paper is to monitor daily health information to manage the dosage adjustment and health care of dementia patients. Measures of the amount of outdoor action and the resulting information on momentum can be health information. The patient profile management system profiles patient's daily information. Patient's daily information can be generated and the disappearance of the patient can be prevented through position information by integrating patient data received via a smart watch. In this paper, a function that analyzes patient's momentum and integrates received data is included to implement such a system.

The amount of exercise analysis calculates the number of steps measured by the acceleration sensor as momentum according to the rules. After the acceleration sensor data received from the smart watch is integrated with data about a patient's sex, age, weight, and height stored in the server, the integrated data generates momentum information.

5.1. Amount of Exercise Analysis. The step count obtained through the step detection algorithm can be used as data that measures momentum. The patient's data, which is basically stored in the server, includes age, height, weight, and personal information and this data is used as the standard for measuring a patient's stride and momentum.

The motion characteristics such as stationariness, walking and running, and information corresponding to moving distance and exercise time are needed in order to calculate the momentum. The moving distance can be measured through the GPS sensor, but it is difficult to measure the exact moving distance due to errors of the GPS sensor and the difference between indoors and outdoors. Therefore, the method that multiplies stride by the number of steps is used to calculate the patient's moving distance in this paper. The stride can be calculated by subtracting 100 from an individual's height, and momentum can be calculated as shown in below.

Amount of exercise

$$
=\text { Amount of energy consumption }(\mathrm{Kcal} / \mathrm{min} * \mathrm{~kg})
$$

$*$ Exercise per minute $(\mathrm{min}) *$ Weight $(\mathrm{kg})$. 

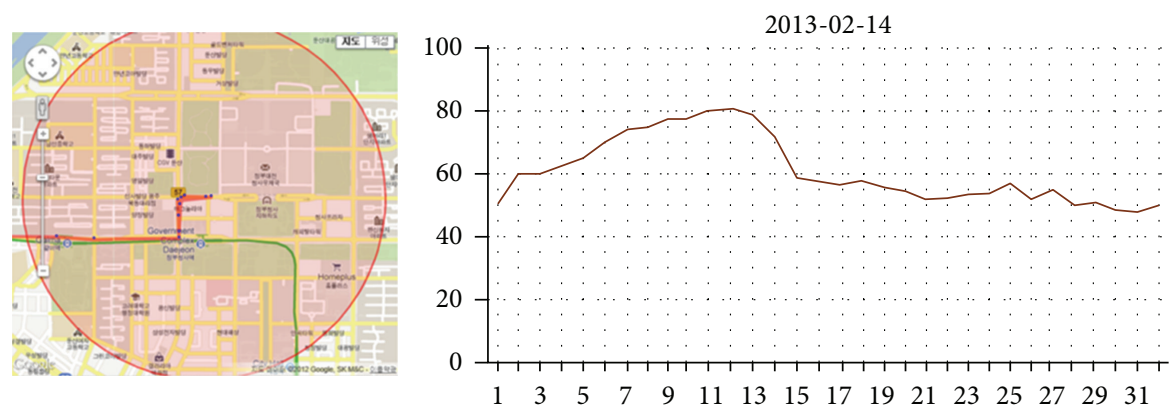

FIGURE 7: Function for monitoring: GPS, amount of activity.
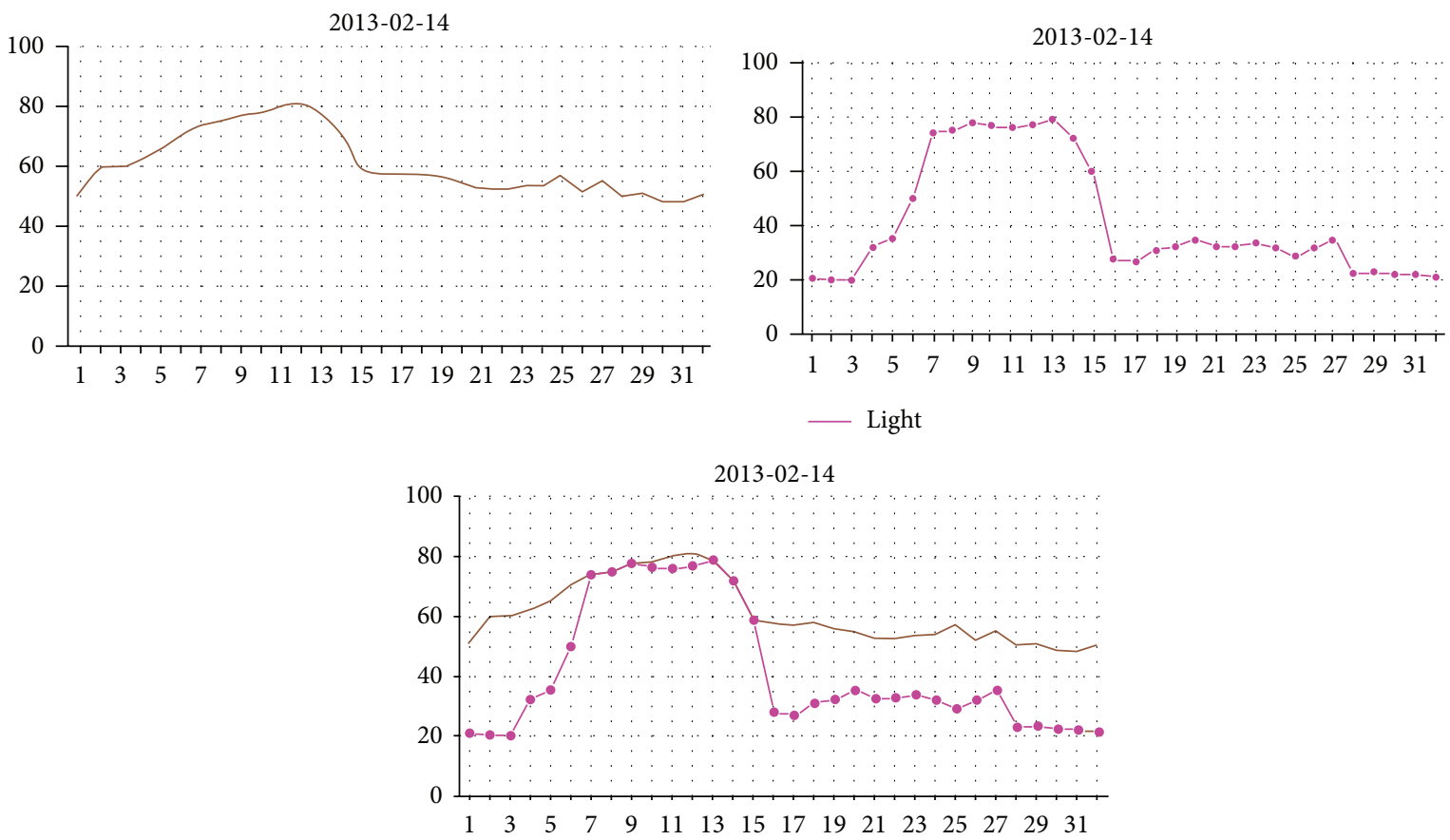

Life information

\section{$41 / 22$}

\begin{tabular}{cccccccccccccccccccccc}
\hline Tic & 1 & 2 & 3 & 4 & 5 & 6 & 7 & 8 & 9 & 10 & 11 & 12 & 13 & 14 & 15 & 16 & 17 & 18 & 19 & 20 \\
\hline Exercise & 51 & 60.2 & 60.3 & 65.4 & 70.9 & 74 & 75 & 77.8 & 78 & 80.4 & 81 & 79 & 72 & 59 & 58 & 57 & 58 & 56.2 & 55.1 & 54 \\
Light & 21 & 20.2 & 20.3 & 32.3 & 35.4 & 50.0 & 73.3 & 77.5 & 78.3 & 76.2 & 76 & 77 & 77.2 & 56 & 28 & 27.2 & 31 & 32.2 & 32.6 & 29.2 \\
Outdoor & $\mathrm{N}$ & $\mathrm{N}$ & $\mathrm{N}$ & $\mathrm{Y}$ & $\mathrm{Y}$ & $\mathrm{Y}$ & $\mathrm{Y}$ & $\mathrm{Y}$ & $\mathrm{Y}$ & $\mathrm{Y}$ & $\mathrm{Y}$ & $\mathrm{Y}$ & $\mathrm{Y}$ & $\mathrm{Y}$ & $\mathrm{Y}$ & $\mathrm{N}$ & $\mathrm{N}$ & $\mathrm{N}$ & $\mathrm{N}$ & $\mathrm{N}$ \\
\hline
\end{tabular}

Figure 8: Patient profile system.

Energy consumption varies with motion characteristics and bottom surface. Table 2 shows energy consumption when walking on the basic asphalt.

The monitoring system developed in this paper can monitor a patient's momentum, current position, and the amount of light through a web page by using the GPS route information during outdoor activities, step detection, and momentum detection. Figure 7 shows the functions of monitoring server.
5.2. Create Patient Profiles. The patient's profile includes the patient's momentum, amount of light, and indoor and outdoor detection information by GPS. The patient's data is obtained in every cycle and the patient's momentum is calculated. The calculated result is integrated and then stored.

Figure 8 shows the screen applying the patient profile management system developed in this paper. The momentum obtained from the patient is divided into momentum, which is converted into a percentage and momentum converted into 
TABLE 1: Experimental results.

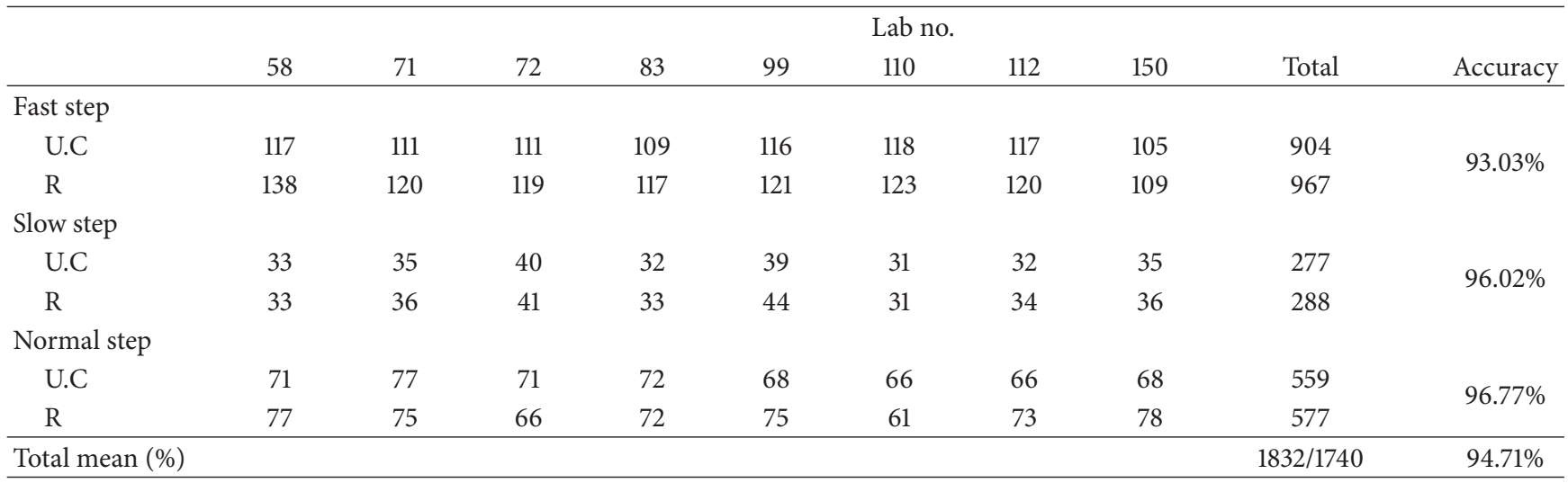

U.C.: user count-The number of steps counted by the user.

$\mathrm{R}$ : result of algorithm-The number of steps counted by the proposed algorithm.

TABLE 2: Amount of exercise on asphalt.

\begin{tabular}{lcccc}
\hline & 1 min & 2 min & 3 min & 10 min \\
\hline $50 \mathrm{Kg}$ & 4 & 8 & 12 & 120 \\
$60 \mathrm{Kg}$ & 3.8 & 9.6 & 14.4 & 144 \\
$70 \mathrm{Kg}$ & 5.6 & 11.2 & 16.8 & 168 \\
$80 \mathrm{Kg}$ & 6.4 & 12.8 & 19.2 & 192 \\
$90 \mathrm{Kg}$ & 7.2 & 14.4 & 21.6 & 216 \\
$100 \mathrm{Kg}$ & 8.0 & 16 & 23 & 240 \\
\hline
\end{tabular}

calories. After being integrated with light data, the profile can be developed of a patient's daily life. The patient's profile is updated daily. And it stores the daily information and moving route measured for a day. If the data is accumulated, the doctor can determine a more exact dosage and treatment method through the patient's daily life data.

\section{Conclusion}

In this paper, we developed an ubiquitous health management system for dementia patients following the concept of IoT. It is composed of a watch-type monitoring device and server that not only monitors patients' locations but also manages patients' health by determining patients' activity according to the data derived with the step detection algorithm, along with the ambient light sensor and accelerometer. According to the results of the experiments, normal steps have $96 \%$ accuracy in detection and on average showed $94 \%$ accuracy.

Typical medical services for dementia focused mainly on tracking the patients' location to prevent a patient from going missing or getting lost. The system developed in this paper provides and monitors the health information of the patients as well as location tracking. Further research based on this work could include a more comprehensive analysis of a patient's activities such as running or sitting and extensive application of the IoT paradigm.

\section{Conflict of Interests}

The authors declare that there is no conflict of interests regarding the publication of this paper.

\section{Acknowledgment}

This research is supported by Seoul R\&BD Program (SS110008).

\section{References}

[1] M. H. Tabert, X. Liu, R. L. Doty et al., "A 10-item smell identification scale related to risk for Alzheimer's disease," Annals of Neurology, vol. 58, no. 1, pp. 155-160, 2005.

[2] M. Brahami, A. Baghdad Atmani, and A. Matta, "Dynamic knowledge mapping guided by data mining: application on healthcare," Journal of Information Processing Systems, vol. 9, no. 1, pp. 1-30, 2013.

[3] Company Keruve, 2008, http://www.keruve.com/.

[4] U-Safe Gangnam, 2009, http://www.gangnam.go.kr/.

[5] KT I-Search, 2009, http://www.kt.com/.

[6] G. E. Mead, W. Morley, P. Campbell, C. A. Greig, M. McMurdo, and D. A. Lawlor, "Exercise for depression," The Cochrane Database System Reviews, vol. 3, Article ID CD004366, 2009.

[7] H. Y. Moon, S. H. Kim, Y. R. Yang et al., "Macrophage migration inhibitory factor mediates the antidepressant actions of voluntary exercise," Proceedings of the National Academy of Sciences of the United States of America, vol. 109, no. 32, pp. 13094-13099, 2012.

[8] B. Kim, T. Kim, H.-G. Ko, D. Lee, S. J. Hyun, and I.-Y. Ko, "Personal genie: a distributed framework for spontaneous interaction support with smart objects in a place," in Proceedings of the 7th International Conference on Ubiquitous Information Management and Communication (ICUIMC '13), Kota Kinabalu, Malaysia, January 2013.

[9] R. S. H. Istepanian, S. Hu, N. Y. Philip, and A. Sungoor, "The potential of Internet of $\mathrm{m}$-health things " $\mathrm{m}$-IoT" for noninvasive glucose level sensing," in Proceedings of the 33rd Annual International Conference of the IEEE Engineering in Medicine and Biology Society (EMBS '11), pp. 5264-5266, Boston, Mass, USA, September 2011. 
[10] C. Doukas and I. Maglogiannis, "Bringing IoT and cloud computing towards pervasive healthcare," in Proceedings of the 6th International Conference on Innovative Mobile and Internet Services in Ubiquitous Computing (IMIS '12), pp. 922-926, Palermo, Italy, 2012.

[11] L. Atzori, A. Iera, and G. Morabito, "The internet of things: a survey," Computer Networks, vol. 54, no. 15, pp. 2787-2805, 2010.

[12] J. Yang, "Toward physical activity diary: motion recognition using simple acceleration features with mobile phones," in Proceedings of the 1st ACM International Workshop on Interactive Multimedia for Consumer Electronics (IMCE '09), pp. 1-10, New York, NY, USA, October 2009.

[13] L. Bao and S. S. Intillem, "Activity recognition from userannotated acceleration data," in Pervasive Computing, vol. 3001 of Lecture Notes in Computer Science, pp. 1-17, Springer, Berlin, Germany, 2004.

[14] J. Baek, G. Lee, W. Park, and B. J. Yun, "Accelerometer signal processing for user activity detection," in Knowledge-Based Intelligent Information and Engineering Systems, vol. 3215 of Lecture Notes in Computer Science, pp. 610-617, Springer, Berlin, Germany, 2004.

[15] N. Ravi, N. Dandekar, P. Mysore, and M. L. Littman, "Activity recognition from accelerometer data," in Proceedings of the 20th National Conference on Artificial Intelligence (AAAI'05), vol. 20, pp. 1541-1546, Pittsburgh, Pa, USA, July 2005.

[16] H. W. Yoo, J. W. Suh, E. J. Cha, and H. D. Bae, "Walking number detection algorithm using a 3-axial accelerometer sensor and activity monitoring," Korea Contents Association Journal, vol. 8, no. 8, pp. 253-260, 2008.

[17] S. H. Shin, C. G. Park, J. W. Kim, H. S. Hong, and J. M. Lee, "Adaptive step length estimation algorithm using low-cost MEMS inertial sensors," in Proceedings of the IEEE Sensors Applications Symposium (SAS '07), pp. 1-5, San Diego, Calif, USA, February 2007.

[18] Y. H. Noh, S. Y. Ye, and D. U. Jeong, "System implementation and algorithm development for classification of the activity states using 3 axial accelerometer," Journal of the Korean Institute of Electrical and Electronic Material Engineers, vol. 24, no. 1, pp. 81-88, 2011.

[19] Y. Luo, O. Hoeber, and Y. Chen, "Enhancing Wi-Fi fingerprinting for indoor positioning using human-centric collaborative feedback," Human-centric Computing and Information Sciences, vol. 3, article 2, pp. 1-23, 2013.

[20] J. Ahn and R. Han, "An indoor augmented-reality evacuation system for the Smartphone using personalized Pedometry," Human-centric Computing and Information Sciences, vol. 2, article 18, pp. 1-23, 2012. 


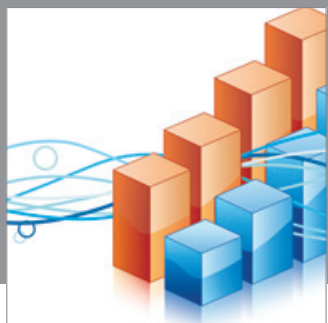

Advances in

Operations Research

mansans

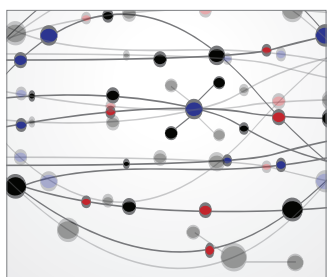

The Scientific World Journal
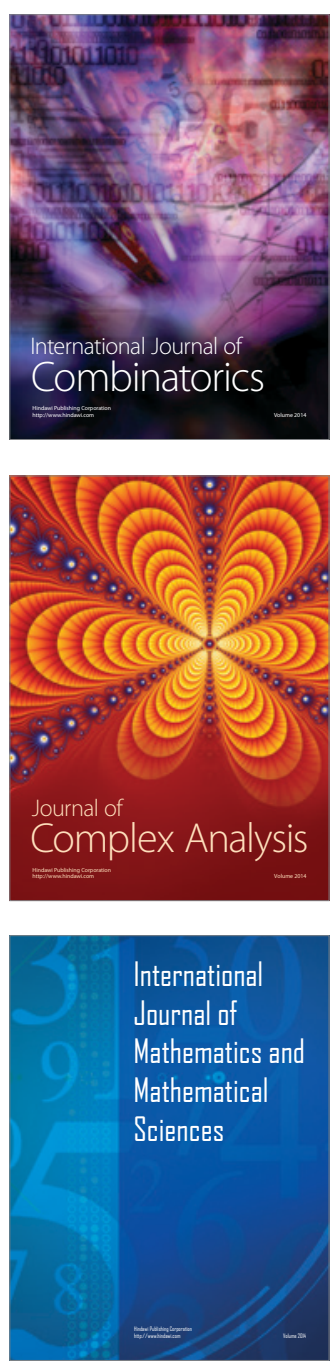
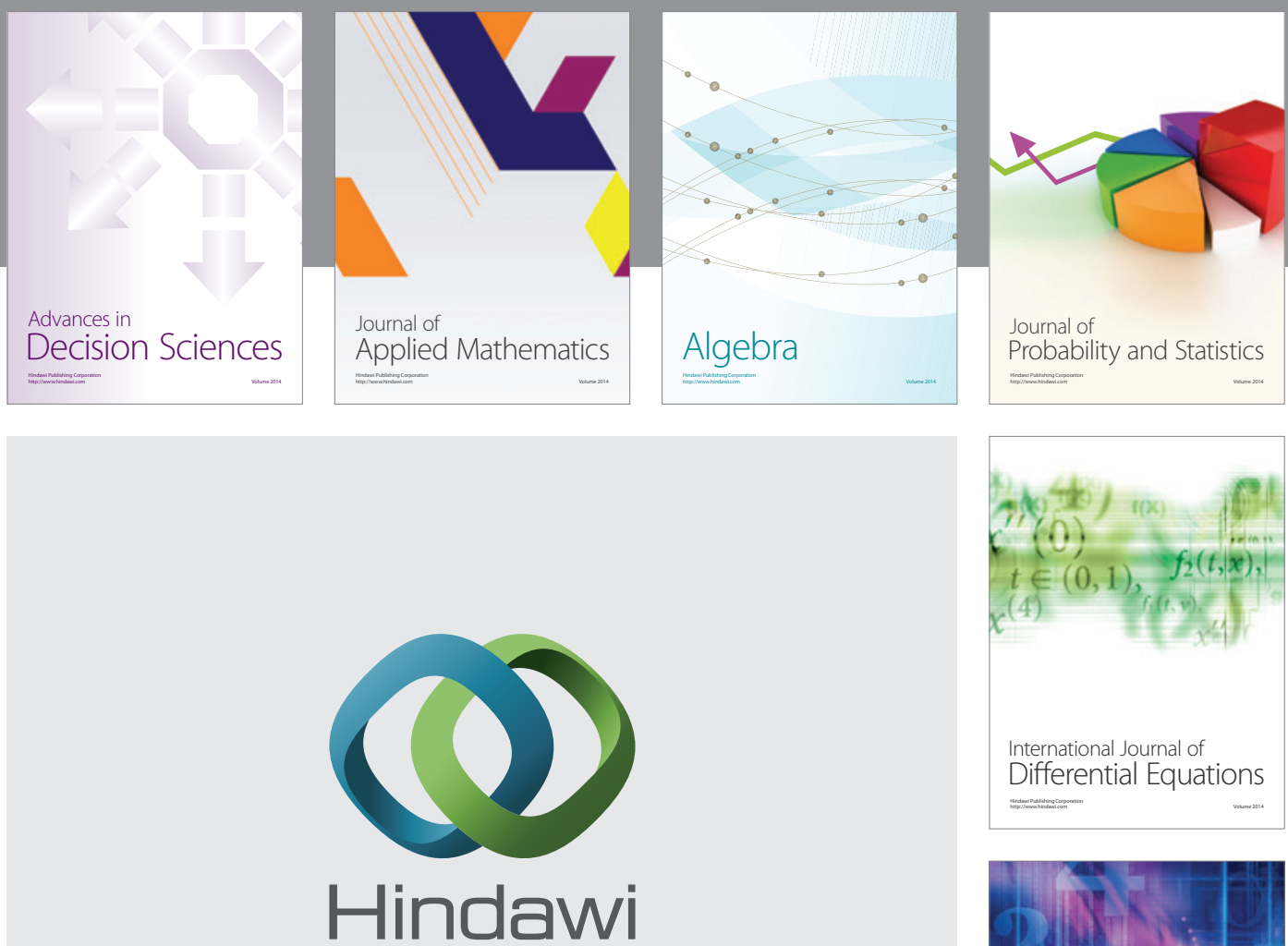

Submit your manuscripts at http://www.hindawi.com
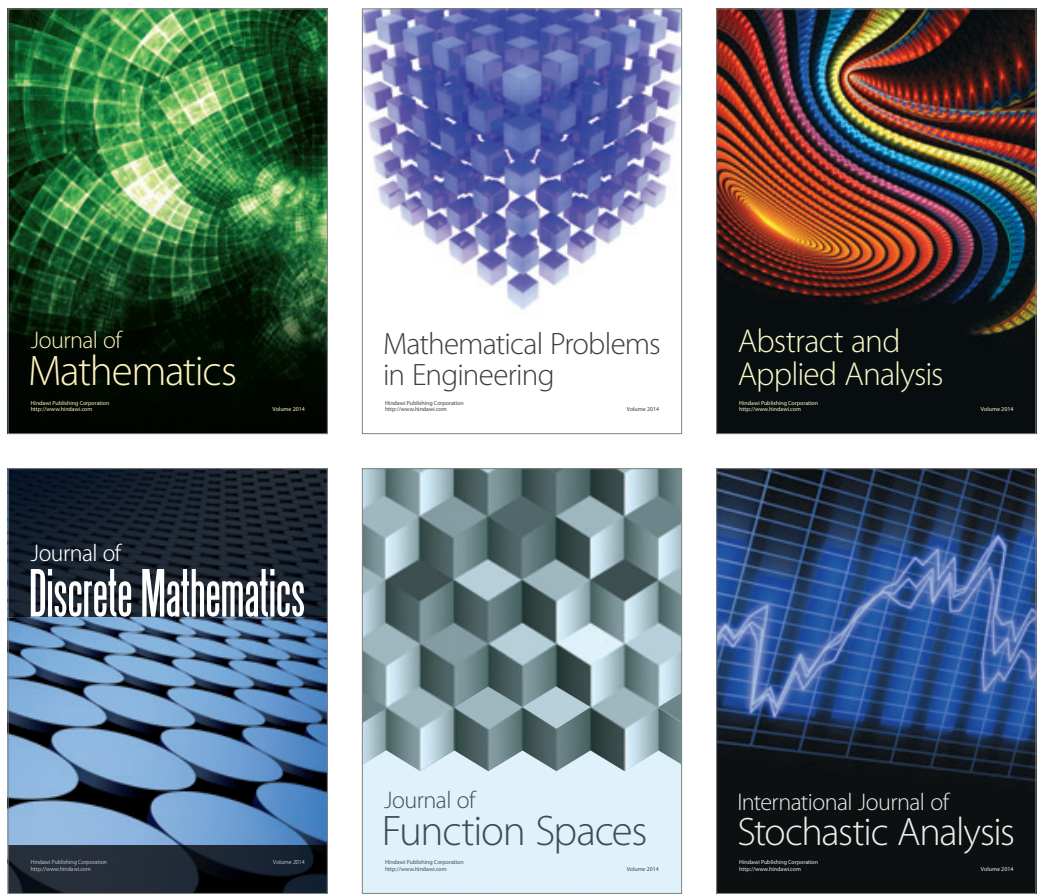

Journal of

Function Spaces

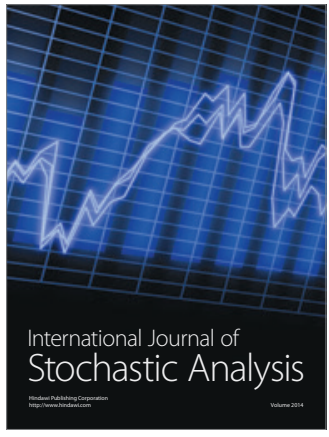

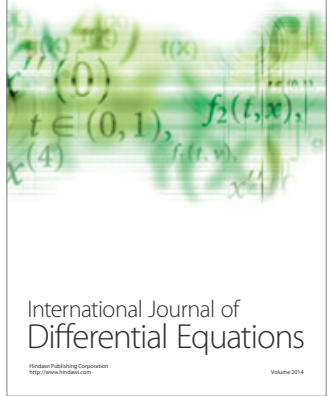
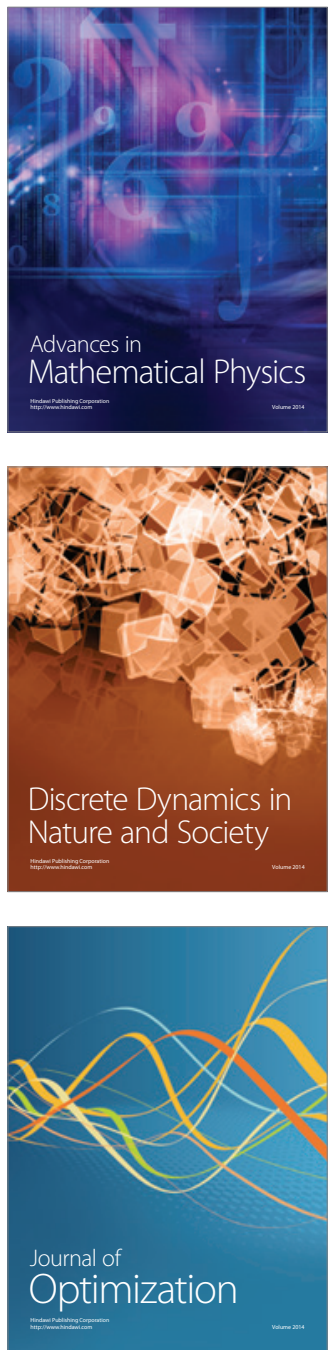